\title{
Transaxillary Endoscopic Breast Augmentation
}

\author{
Hyung-Bo Sim \\ BR Baram Clinic, Seoul, Korea
}

The axillary technique is the most popular approach to breast augmentation among Korean women. Transaxillary breast augmentation is now conducted with sharp electrocautery dissection under direct endoscopic vision throughout the entire process. The aims of this method are clear: both a bloodless pocket and a sharp non-traumatic dissection. Round textured or anatomical cohesive gel implants have been used to make predictable welldefined inframammary creases because textured surface implants demonstrated a better stability attributable to tissue adherence compared with smooth surface implants. The axillary endoscopic technique has greatly evolved, and now the surgical results are comparable to those with the inframammary approach. The author feels that this technique is an excellent choice for young patients with an indistinct or absent inframammary fold, who do not want a scar in the aesthetic unit of their chest.

Keywords Axilla / Endoscopes / Mammaplasty / Breast implants

\author{
Correspondence: Hyung-Bo Sim \\ BR Baram Clinic, 5 Nonhyeon-ro \\ 155-gil, Gangnam-gu, Seoul 135-892, \\ Korea \\ Tel: $+82-2-511-8758$ \\ Fax: +82-2-540-8759 \\ E-mail:wsimww@gmail.com
}

No potential conflict of interest relevant to this article was reported.

\section{INTRODUCTION}

The transaxillary approach for breast augmentation was first reported in the 1970s and has been developed as a useful method; however, as a blind technique, it had limitations compared with the inframammary or periareolar approach. These included possible hematoma, trauma, and inability to predict the extent of dissection or produce a discrete inframammary crease. To address these drawbacks, the performance of the transaxillary approach for breast augmentation with the use of an endoscope has evolved [1-3]. In the initial stage of the use of the endoscopic approach, the traditional blind technique was blended with the endoscope in the process of surgery. As a result, less bleeding and more precise dissection was sometimes achieved compared to the traditional techniques; however, tissue damage from blunt dissection was still inevitable. In addition, the incomplete dissection of the costal origin of the pectoralis major muscle or the inferomedial part of the pocket could cause a higher incidence of upward or outward displacement of the implant, or inconsistency or asymmetry of the inframammary crease as a result of reconnecting the muscles [1].

Transaxillary breast augmentation is now conducted with sharp electrocautery dissection under direct endoscopic vision throughout the entire process. The benefits of this method are clear: both a bloodless pocket and a sharp non-traumatic dissection. The costal origin of the pectoralis major is completely divided to create a dual plane [4]. The precise well-fit dimensions of the pocket are prepared, and round textured or anatomical cohesive gel implants are used.

This technique has evolved enough to facilitate the patient's quick recovery and early abatement of pain, the reduction of the need for drainage, reduced displacement of implants, and the securing of a well-defined and symmetrical inframammary crease. Thus the outcomes of endoscopic transaxillary breast augmentation are now comparable to those with the inframammary approach. 


\section{METHODS}

\section{Objective of study}

This study included 232 female recipients who underwent endoscope-assisted transaxillary dual plane breast augmentation from December 2006 to December 2012. The mean patient age was 28.5 years, with a range from 23 to 48 years of age. The average height and weight of the patients were $164.5 \mathrm{~cm}$ and 50.3 $\mathrm{kg}$, respectively. Most of them had small breasts with indistinct inframammary creases and had not shown signs of ptosis, and none of the patients had a constriction in the bottom part of the breast. 188 round textured cohesive gel implants and 44 anatomical gel implants were used (Table 1).

The size of the implants ranged from 203 to $440 \mathrm{~mL}$ with a mean size of $261 \mathrm{~mL}$. Closed suction drainage was not inserted into any of the patients. The follow-up ranged from 6 to 36 months (mean, 11 months). The indications for the transaxillary approach include the patient's desire, $3 \mathrm{~cm}$ or less in areola diameter, or the patient's choice of location of the scars after the doctor had explained the various approach methods.

\section{Instrument}

An endoscope with a tubing work space was used (Richard Wolf GmbH, Knittlingen, Germany) (Fig. 1). A straight needle

\section{Table 1. Implant types}

\begin{tabular}{|lcrc|}
\hline Implant type & \multicolumn{1}{c}{ Textured $(\mathbf{n}=\mathbf{2 3 2})$} & Smooth \\
\hline Round & Allergan & 188 & - \\
Anatomical & Mentor (CPG) & 20 & - \\
& Polytech (Replicon) & 24 & - \\
\hline
\end{tabular}

tip and curved electrocautery devices were used for dissecting the pocket, and the bleeding was controlled by bipolar forceps. Inside the tubing work space, the cannula could move freely and safely, having less chance of penetrating between the ribs.

\section{Surgical method}

All of the procedures were performed under general anesthesia. Each patient was lying on her back with her arms extended to 90 degrees. The preoperative evaluations of all of the anatomical landmarks were checked, and in the deepest part of the armpit, the incision line was marked, anteriorly $1 \mathrm{~cm}$ away from the lateral border of the pectoralis major muscle to keep the posterior incision end high in the axilla, about $4 \mathrm{~cm}$ in length. The skin was incised until the subcutaneous fat was exposed. From here, shallow subcutaneous dissection proceeded $3 \mathrm{~cm}$ toward the lateral border of the pectoralis major muscle.

\section{Armpit pocket (part 1 in Fig. 2)}

Under direct vision, the pectoral fascia was reached. The pectoral fascia opened and the layer between the pectoralis major and minor muscle was accessed. In this process, hemostasis for the lateral thoracic vessels was necessary. It should be noted that, especially to protect the intercostobrachial nerve and medial brachial cutaneous nerve, surgeons should pay attention to avoid damage to the axillary fat pad. When the clavipectoral space was ready through the axillary window, the endoscope (Richard Wolf GmbH) (Fig. 1) with a tubing work space was introduced and the subpectoral sharp dissection began. The sequence of the pocket dissection was conducted from the superomedial, down to the inferomedial, the inferolateral, and up to the lateral parts in a clockwise fashion on the right breast (Fig. 2). The ap-

\section{Fig. 1. Tubing work space endoscopic equipment}

(A) Endoscope (Richard Wolf GmbH) with a tubing work space. (B) A needle tip cautery, a curved cautery cannula, and a bipolar forceps (Richard Wolf).
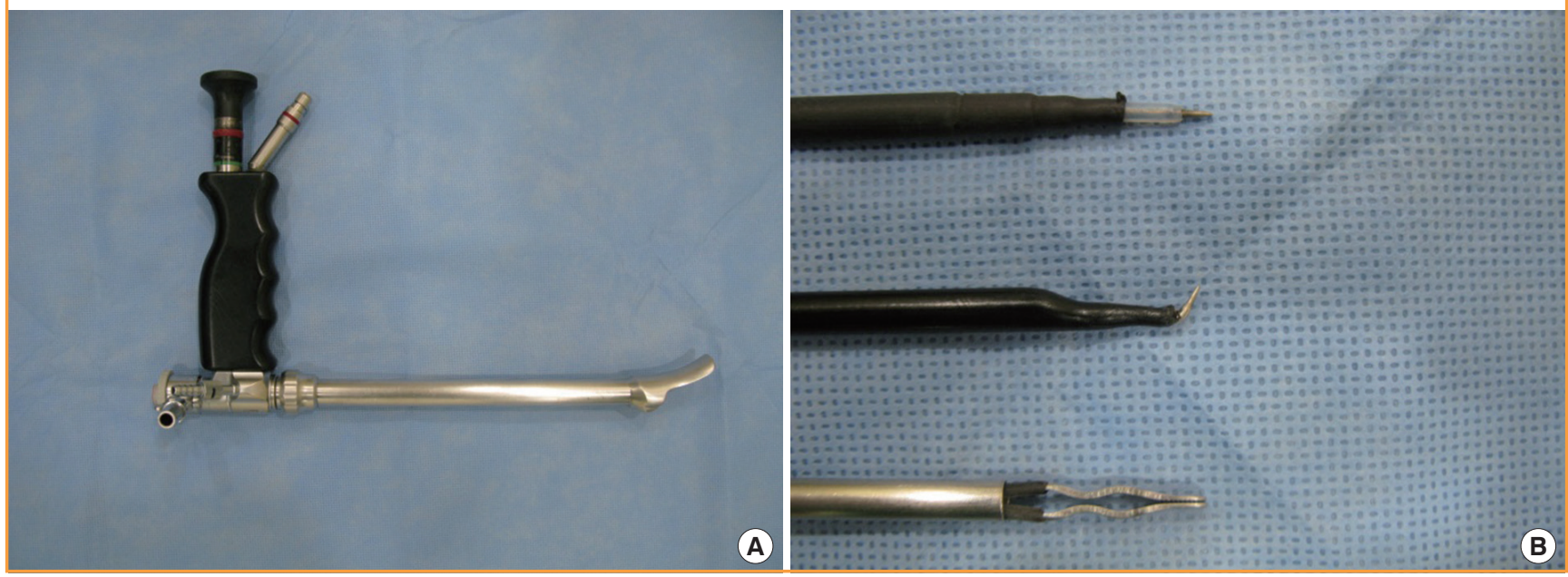


\section{Fig. 2. The order of sequential dissection}

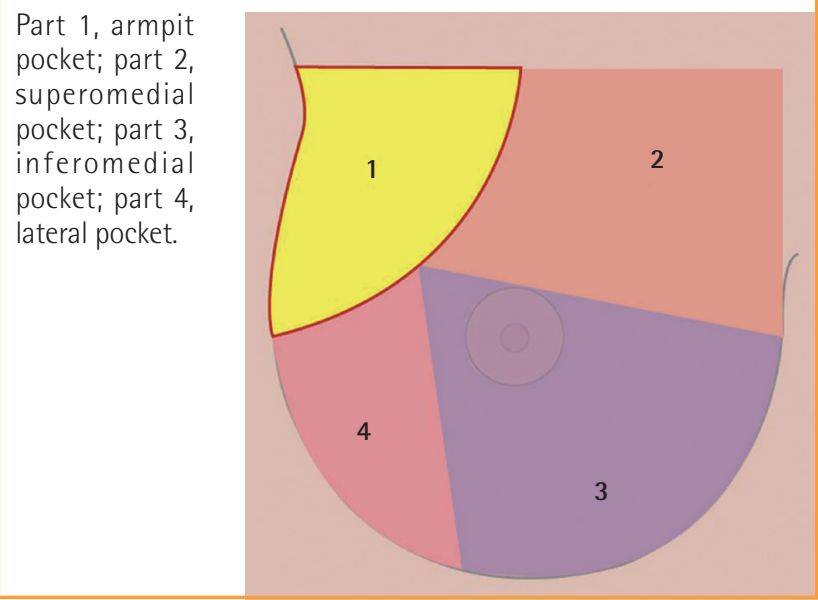

\section{Fig. 3. Transition from part 1 to part 2}

Loose areolar tissues between the pectoralis muscles and thoracoacromial fat pad.

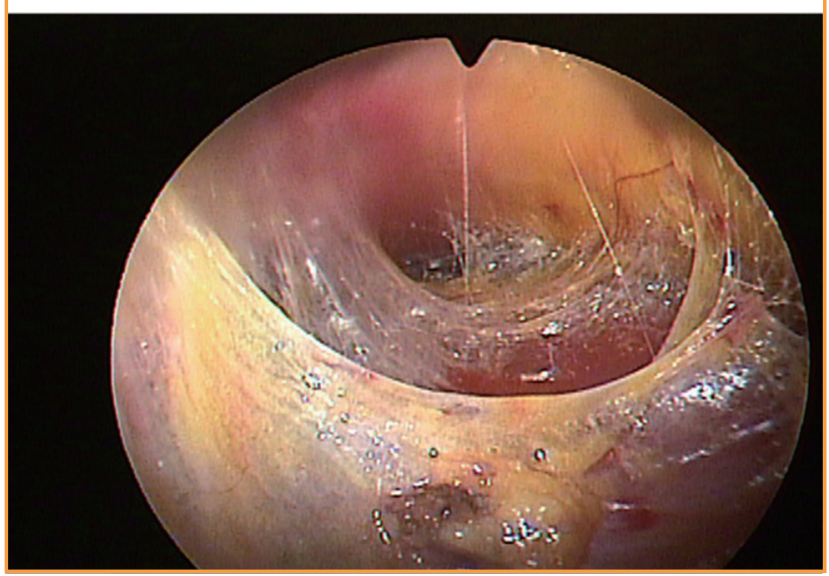

plication of blunt equipment was abandoned, and only sharp electrocautery dissection was executed for the entire process under direct endoscopic vision. When using an endoscope, the mechanical damage to the ribs and their cartilage was minimized by trying not to touch them to prevent bleeding. If necessary, the preoperative marks were pressed with a finger or the needle through the skin during surgery to confirm that the pocket was in the appropriate location as planned.

\section{Superomedial pocket (part 2 in Fig. 2, Fig. 3)}

After confirming the loose areolar tissues between the pectoralis major and minor muscle, the endoscope proceeded parallel to the clavicle toward the midsternum. The endoscope was moved forward or backward gradually; thus, the bleeding points could be controlled completely. The discrimination between the white tendinous accessory pectoralis origin from the main body of the sternal origin was confirmed. The accessory slips were di-

\section{Fig. 4. Part 3, division of the costal origins}

Divided and retracted pectoral muscle edges exposing a glistening fascia in frontof yellow subcutaneous fat

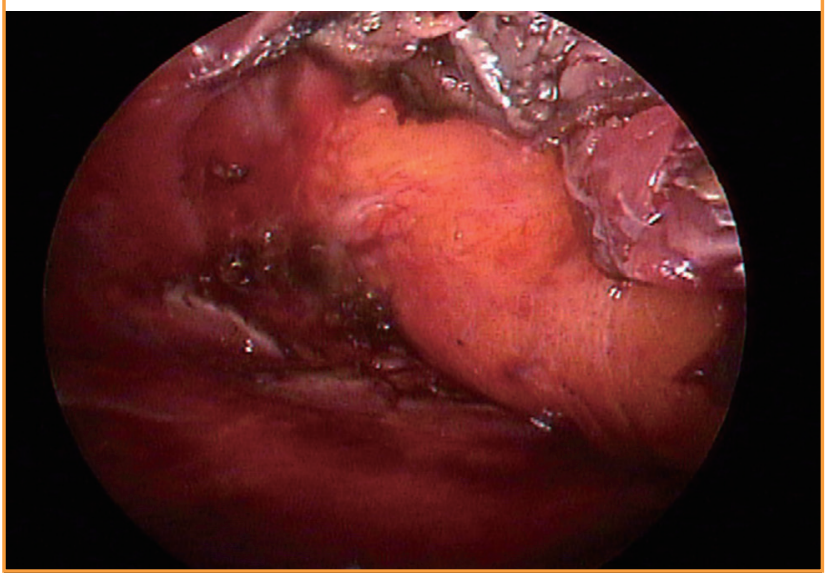

vided completely, and the dissection continued until the main body of the sternal origin had been reached. For the superior boundary, the dissection was controlled not to exceed the range of the thoracoacromial artery. The intermammary distance should be $3 \mathrm{~cm}$ or more to prevent against excessive dissection of the medial side.

\section{Inferomedial pocket (part 3 in Fig. 2, Figs. 4, 5)}

The endoscope was moved from the superomedial pocket to the inferomedial pocket. The sharp penetrating dissection started from the sternocostal junction at the level of the inferior areolar margin, and at first, the costal origin was penetrated with needle tip electrocautery under magnified vision preserving the glistening pectoral fascia just in front of the yellow subcutaneous fat. From medial to lateral, the costal origin was completely divided by electrocautery, and the muscle stumps were coagulated. The author took care to ensure to the hemostasis of the perforators of the internal mammary artery, which are distributed near the inferomedial sternum. The division of the costal origin of the pectoralis major muscle was done $1 \mathrm{~cm}$ off the chest wall to allow for easier hemostasis and minimize the mechanical damage to the costochondrium. In this process, the surgeon should pay careful attention because the costal origin of the pectoralis major consists of several layers across the 4th and 5th rib. Often for patients with high inframammary folds, the dissection was continued downward until the superficial layer of the deep fascia or the rectus fascia had been reached. In those cases, it was important to maintain the thickness of the envelope so that the dissection could proceed under the deep fascia.

It is common to find the abdominal head of the pectoralis origin in a transaxillary endoscopic approach. Laterally to the costal origin, a separate broad band of thin muscle is usually noticed 


\section{Fig. 5. Abdominal head of the pectoralis major}

(A) Laterally at the end of the Part 3 dissection. $(B, C)$ It is common to find the abdominal head of pectoralis origin, which should be divided completely.
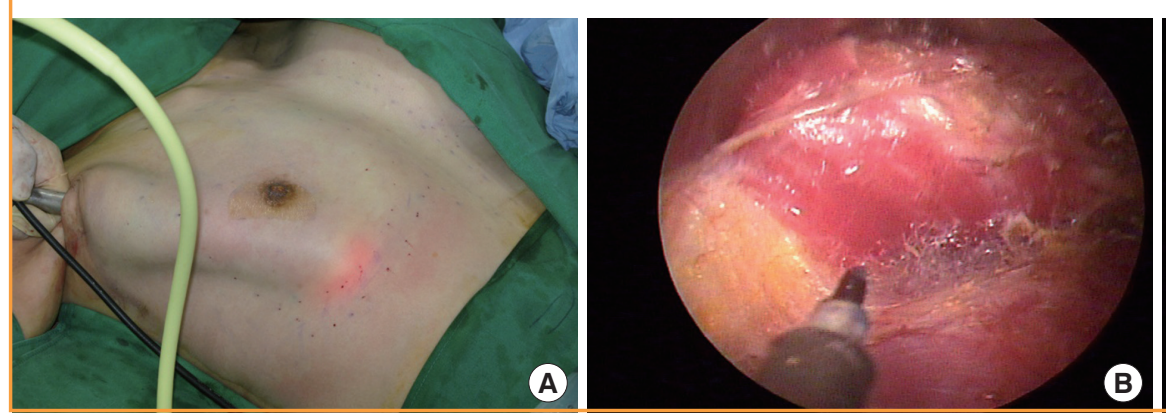

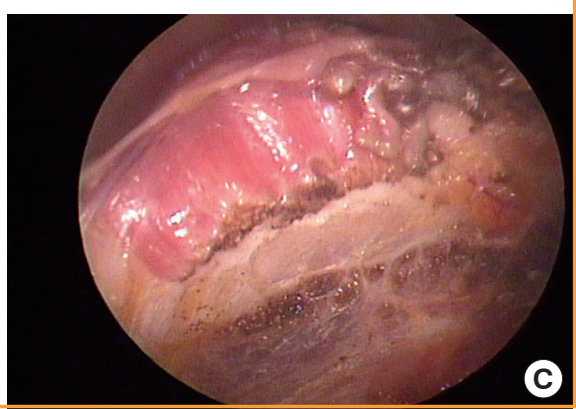

Table 2. Summary of complications

\begin{tabular}{|lc|}
\hline Complications & No. of patients $(\%)(\mathbf{n}=\mathbf{2 3 2})$ \\
\hline Capsular contracture, grade & $5(2.2)$ \\
Bilateral & 1 \\
Unilateral & 4 \\
Delayed seroma & $2(0.9)$ \\
Round & 1 \\
Anatomical & 2 \\
Hematoma & $1(0.4)$ \\
Round & 1 \\
Infection & 0 \\
Total & $8(3.4)$ \\
\hline
\end{tabular}

pocket had to be constructed to fit the planned implant dimensions well. Usually the goal was to create a bloodless pocket ready for the breast implant. The pockets were irrigated with an antibiotic solution. After testing disposable sizers, round or anatomical implants were inserted. The patient was positioned in a semi-Fowler's position, and the breasts' shape, size, and symmetry were checked again. Additional correction was done if necessary. A drainage tube was not used. The subcutaneous layers and skin were closed. An ace bandage was applied to the armpit area with light dressing.

\section{RESULTS}

Among all of the patients, there were no major complications including severe bleeding, infection, breast implant rupture, or severe asymmetry reported or found during the follow-up. There were no pneumothorax nor instrument-related skin burns. No patients reported severe deformation of implants due to the contraction of the pectoralis major muscle or displacement of the implants after the operation. A total of 5 (2.16\%) patients had shown capsular contracture; bilaterality of the contracture was found in 1 patient; unilateral contracture was found in 4 patients; and all of them had a third-degree Baker classifica-

\section{Insertion of implants and wound closure}

When the overall pocket had been prepared, the bleeding areas were rechecked with an endoscope. The size and shape of the 


\section{Fig. 7. Case 1, preoperative and postoperative views}

(A) A 28-year-old woman with bilateral breast hypotrophy. (B) Postoperative views 1 year after the endoscopic transaxillary breast augmentation; $290 \mathrm{~mL}$ textured round cohesive gel implants (Allergan, style 115) were used in both sides.
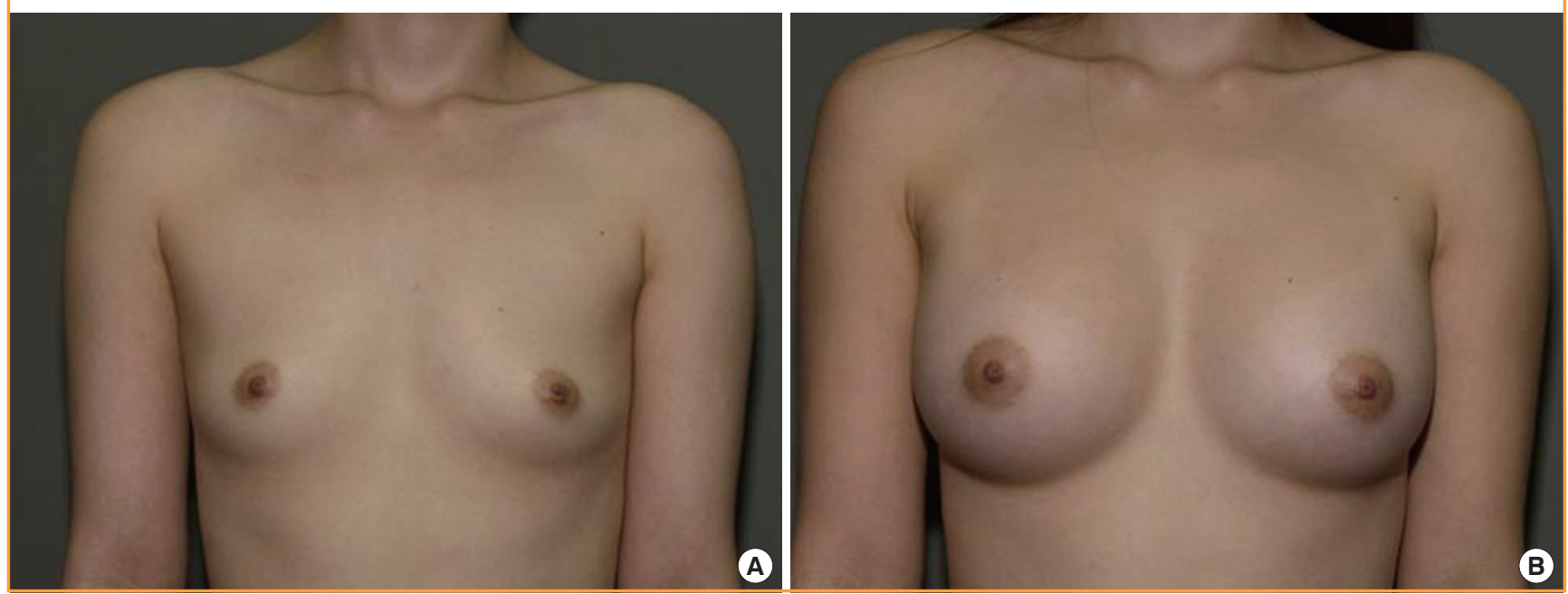

Fig. 8. Case 2, preoperative and postoperative views

(A) A 32-year-old woman with bilateral hypoplastic breasts. (B) Postoperative views 2 years after the endoscopic transaxillary breast augmentation; $322 \mathrm{~mL}$ textured round cohesive gel implants (Allergan, style 115) were used in both sides.

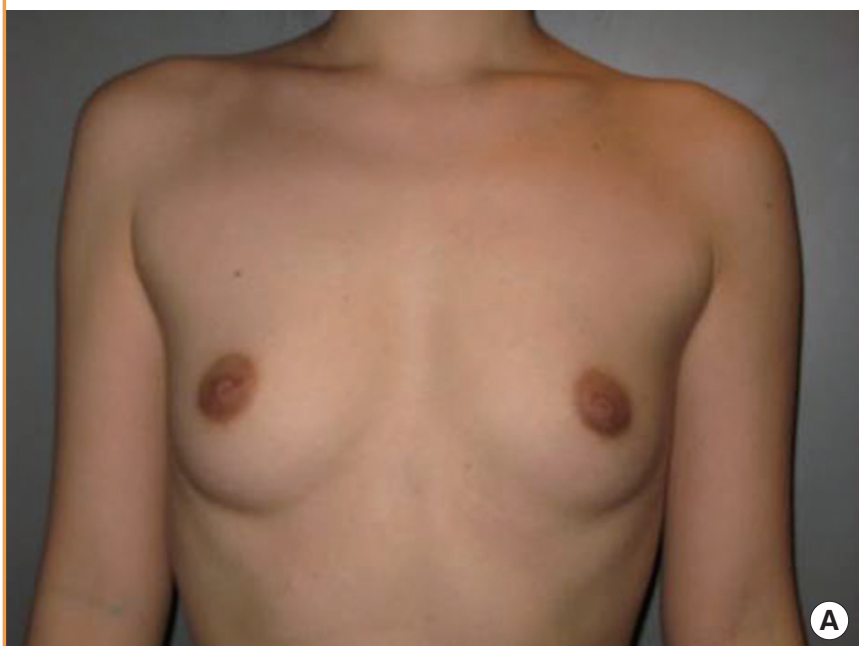

tion. No contracture was found among the patients with anatomical implants. There was no double-bubble deformity or abnormality of the inframammary crease reported. There were 2 cases of unilateral delayed seroma 7 months and 9 months after the surgery, which were managed by replacement with smooth implants. One case of unilateral hematoma occurred 3 weeks after the surgery following playing golf against the doctor's advice (Table 2).

About $84 \%$ of the patients (174 among 232) were able to return to their normal life and work within 3 days of surgery; and all of the patients were able to return to their normal life within 7 days of surgery without any specific symptoms (Figs. 7-9).

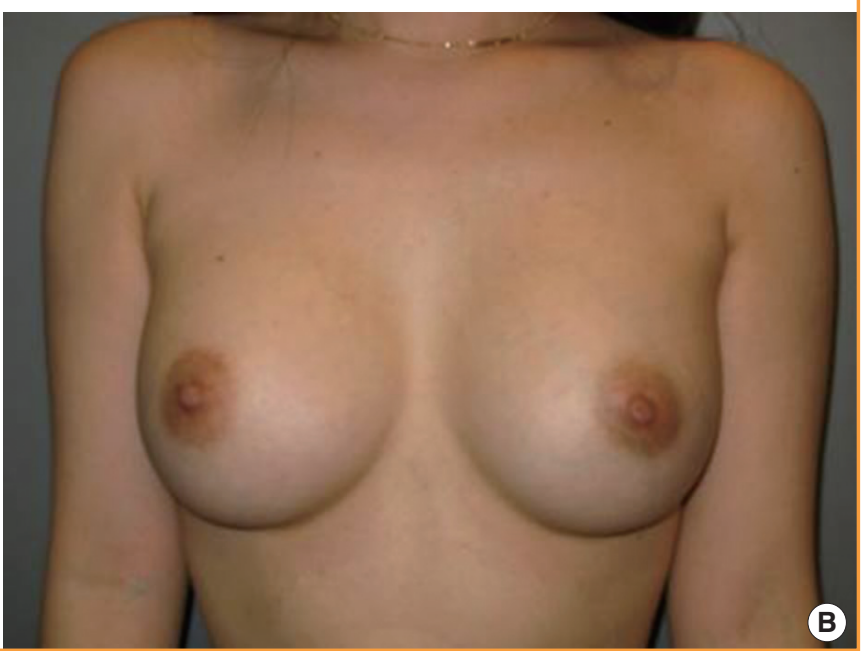

\section{DISCUSSION}

The transaxillary breast augmentation described by Hoehler in 1973 was a kind of partial subpectoral breast augmentation, in which one third of the upper implant was placed under the pectoralis major and two thirds of the implant was placed over the rectus sheath and external oblique muscle. When compared to the inframammary or periareolar approach, it has the benefits of not leaving any scars in the aesthetic unit of the breast or any mechanical disturbance of the scar maturation due to the implant or external forces such as massage.

However, from the early stages of the axillary approach, the disadvantages of the blind technique, mainly including difficult he- 


\section{Fig. 9. Case 3, preoperative and postoperative views}

(A) A 38-year-old woman with bilateral involutional hypoplasia. (B) Postoperative views 1 year after the endoscopic transaxillary breast augmentation; $280 \mathrm{~mL}$ anatomical implants (Mentor, CPG321) were used in both sides.

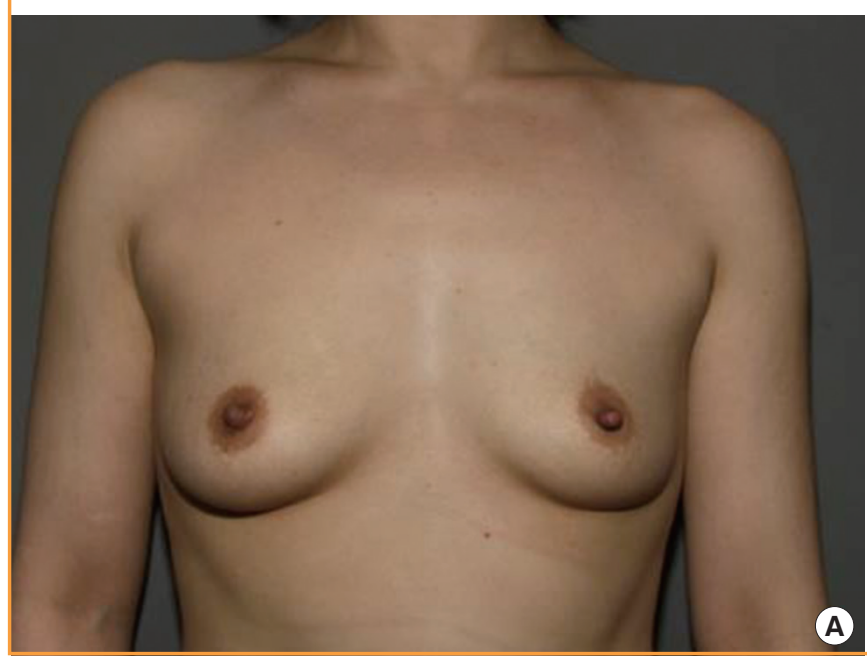

mostasis and traumatic dissection, and difficulty in creating a precise pocket and making symmetrical inframammary creases, had been reported. Although there are relatively few blood vessels or perforators in the space between the pectoralis major muscle and pectoralis minor muscle, under the blind method, damage to the perforators inside the pectoral muscle was inevitable during dissection of the costal origin, the perforating vessels near the sternal border, and the fourth intercostal neurovascular bundle. In addition, when bleeding occurred, there were only a few ways to perform hemostasis including manual pressure or irrigation, and the blood-stained tissue might have added to the risk of capsular contracture. Also, with the blind technique, it was possible that the dissection plane would be incorrect, accidentally being created either under the pectoralis minor muscle or above the pectoralis major muscle. It was also possible to lift the pectoralis major muscle connected with the external oblique muscle and its fascia, and the serratus anterior muscle as one layer [6]. The inframammary crease might not have been smooth and could have been irregular due to the incomplete division of the costal origin and the difficulty of making an even discrete line for the creases. In the study of Tebbetts [1], the difficulty of creating adequate pocket dimensions with the blind technique was mentioned, and a considerable degree of strength was required when dissecting the origin of the pectoralis major blindly. The most common resulting complications were imbalance or migration of the inframammary fold. The limitation of a blind technique for patients who have ptotic breasts but want transaxillary breast augmentation was also explained. About $8.6 \%$ of patients experienced displacement of the implant upward after blind surgery in this study [7]. Based on the various studies reporting that

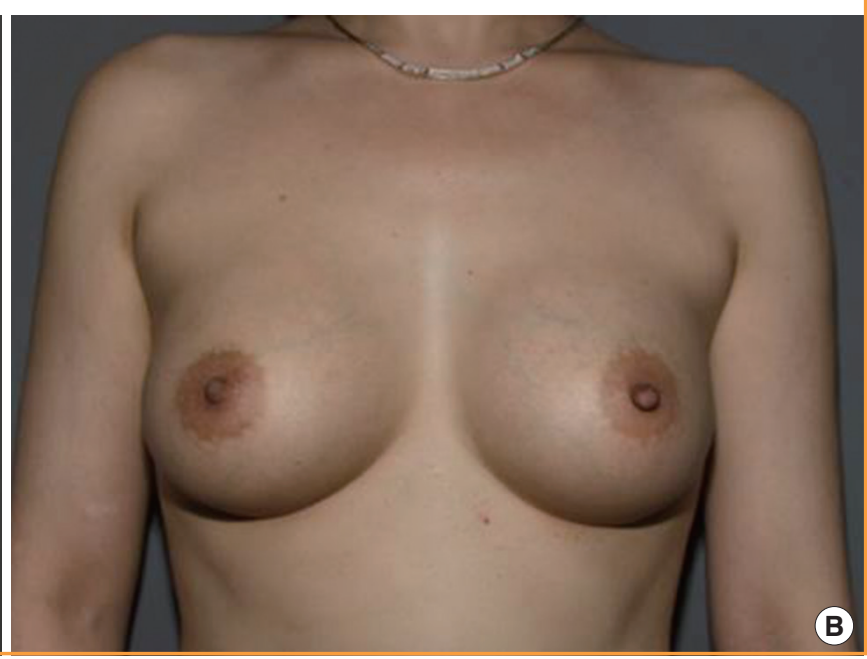

hematoma is an important factor in capsular contracture, it is certain that the blind approach has a higher risk of capsular contracture. Several studies have shown that capsular contracture was reduced with the use of an endoscope [8-11].

Endoscope-assisted transaxillary approach breast augmentation was first introduced by Ho [2] in 1993, 20 years after the advent of the blind transaxillary approach. Many additional studies followed; however, in most of the cases, the dissection was conducted with blunt equipment, and the endoscope was focused on the control of hemostasis and the confirmation of the pocket location $[3,11,12]$. In medial and lateral dissection, several reports $[2,3,11]$ have suggested blunt dissection rather than using electrocautery under the endoscope. However, the blind technique did not guarantee complete hemostasis, an accurate pocket size and location, a precise plane, a smooth consistent symmetrical inframammary crease, or any other benefit. The author did not use any blunt dissection throughout the entire procedure and actively used sharp electrocautery dissection even in the medial and lateral dissection. However, if the dual plane procedure was not performed correctly with incomplete division of the costal origin, considerable complications could occur including high riding of the implant and inconsistent asymmetrical inframammary creases $[4,10]$.

Dual plane breast augmentation [4] overcame the disadvantages of both partial and total subpectoral breast augmentation surgery. Its advantage is to take appropriate actions in each circumstance by changing the surgical method according to the extent of attachment between the breast tissue and the pectoralis muscle. The dual plane can prevent superolateral displacement of implants and control the bulge of the superior and me- 
dial part of the breast. Bending or malposition, which is possible during the contraction of the pectoralis major muscle, can also be reduced. The method conducted in this study was to completely separate the costal origin of the pectoralis major muscle without dissecting between the pectoralis major muscle and the breast tissue (Dual plane I). Results similar to those of Tebbetts [4], who reported that the pectoralis major muscle can move about 2 to $4 \mathrm{~cm}$ upward with the division of the costal origins, were possible to reproduce.

Dissection of the medial pocket requires extra attention when approaching the central part of the sternum. Unless the sternal origin of the pectoralis main body is preserved, window shading or a synmastia can occur. In addition, the surgeon must be cautious about the location of the internal mammary artery perforator. However, to reduce the lateral displacement of the implant and keep the medial volume of the breast, it is important to secure an appropriate medial pocket [4]. In a study based on anatomical considerations, it was noted that the most medial part in the sternal origin of the petoralis major is composed of an arc of about 1 inch of the median raphe from the sternocostal junction; thus, this could be evident that the medial pocket could be dissected close to the lateral sternum without any problems [13].

Tebbetts [14] reported that prospective hemostasis and exclusion of blunt equipment allowed for returning to normal activities within 24 hours after the operation. Although the degree of pain and the discomfort with activities are subjective, reduction of pain and shortening of the recovery period could be reproduced with sharp electrocautery dissection in this study.

The disadvantage of traditional transaxillary breast augmentation was the possibility of nerve and lymphatic damage in the armpit area. To minimize the damage to the intercostobrachial nerve and medial brachial cutaneous nerve, the lateral edge of the pectoralis major muscle should be approached through thin subcutaneous dissection after the incision, avoiding any dissection of the axillary fat pad. In addition, lymph damage may be caused during any of the surgical steps, and this can disturb the diagnosis of breast cancer and its staging. Efforts to address this problem have been emphasized; however, a clear solution has not yet been found $[10,15]$.

It is important to establish a dissection sequence when using an endoscope during surgery (Fig. 2). The reasons are, first, to acquire a sufficient visual field and obtain prompt access to be able to control bleeding. After the hemostasis of the medial pocket, the zone of greater vascularity, it would be easier to move to the lateral pocket. The second reason is to standardize and limit the motion of the endoscope to reduce tissue damage and bleeding. The third is to minimize any unnecessary motions by conducting the surgery according to the planned order to shorten the operation time.

In this study, the author used both textured round and anatomical implants rather than smooth surface implants. In a transaxillary breast augmentation, it is difficult to determine the location of the new inframammary crease and set the new crease in a planned position compared with an inframammary approach. Smooth surface implants have tended to behave unpredictably and migrate downwards. However, textured surface implants have demonstrated a better stability, attributable to tissue adherence. In addition, the results of the meta-analysis showed that capsular contracture occurred approximately five times more frequently with smooth surface implants than with textured surface implants [16].

Because of the characteristics of endoscopic surgery, several disadvantages have been reported, including the need for special equipment and a learning curve. In addition, the duration of surgery might be longer than with the blind technique. When using the endoscope, the view inside is not realistic, but magnified and distorted images transferred from the camera attached to the endoscope might differ from the actual anatomical figure; the orientation of the camera should also be maintained continuously. Adding to this, the difficulty may be doubled because of the characteristics of the surgery, in that the surgeon should be skilled at creating a dual plane [10].

However, actually, compared to the blind technique, similar or less time was reported to be required for a skilled endoscopic surgeon $[2,11]$. Therefore, the time taken to conduct the surgery is more related to experience with various aspects of the operation. Other disadvantages of the transaxillary approach include that reoperation after capsular contracture might not be possible, and an additional incision site could be added. The thickness of the patient's soft tissue should be considered for dual plane surgery. In principle, if the skin thickness of the inframammary crease is less than $4 \mathrm{~mm}$ in the pinch test, dual plane surgery cannot be conducted. Usually the axillary approach is not recommended with glandular ptosis, lower pole constriction, or any type of reoperation [10].

Some authors have insisted that transaxillary incision has shown a higher rate of capsular contracture $[17,18]$. However, most of their major operations were conducted through the inframammary approach, and they were not experienced with the transaxillary technique. Furthermore, the sample sizes of these studies were too small and the follow-up time was too short. The primary causes of capsular contracture are subclinical infection and hematoma. Therefore, surgeons should continue to minimize any potential sources of contamination and do their best to create a bloodless pocket and reduce the chance of trauma.

The axillary technique is the most popular approach to breast 
augmentation among Korean women. The East Asian skin complexion tends to leave visible scars, and most young East Asian women are also slim and thin without much breast tissue. In the author's practice, over $60 \%$ of breast augmentations have been performed through the axilla.

The axillary method is a valid incisional approach for breast augmentation if the surgeon has sufficient anatomical knowledge and experience with endoscopic equipment. The axillary endoscopic technique has greatly evolved, and now the surgical results are comparable to those with the inframammary approach.

\section{CONCLUSIONS}

Transaxillary breast augmentation has been conducted with sharp electrocautery dissection under direct endoscopic vision throughout the entire process. The benefits of this method were clear: both a bloodless pocket and a sharp non-traumatic dissection. Attention was turned to minimizing the tissue damage by means of sharp dissection, shortening the period of recovery, relieving pain, and reducing the risk of capsular contracture. The author divided the costal origin of the pectoralis major muscle completely to produce the dual plane type I, which could reduce the chance of displacement or malposition of the implant, and achieved a definite inframammary fold. The author feels that this technique is an excellent choice for young patients with an indistinct or absent inframammary fold, who do not want a scar in the aesthetic unit of their chest.

\section{REFERENCES}

1. Tebbetts JB. Transaxillary subpectoral augmentation mammaplasty: long-term follow-up and refinements. Plast Reconstr Surg 1984;74:636-49.

2. Ho LC. Endoscopic assisted transaxillary augmentation mammaplasty. Br J Plast Surg 1993;46:332-6.

3. Price CI, Eaves FF 3rd, Nahai F, et al. Endoscopic transaxillary subpectoral breast augmentation. Plast Reconstr Surg 1994;94:612-9.

4. Tebbetts JB. Dual plane breast augmentation: optimizing implant-soft-tissue relationships in a wide range of breast types. Plast Reconstr Surg 2001;107:1255-72.

5. Sim HB, Hwang K, Huan F, et al. Anatomy and tensile strength of the abdominal head of the pectoralis major muscle in relation to transaxillary breast augmentation. Aesthetic Plast Surg 2013;37:359-63.
6. Troilius C. Total muscle coverage of a breast implant is possible through the transaxillary approach. Plast Reconstr Surg 1995;95:509-12.

7. Howard PS. The role of endoscopy and implant texture in transaxillary submuscular breast augmentation. Ann Plast Surg 1999;42:245-8.

8. Fryzek JP, Signorello LB, Hakelius L, et al. Local complications and subsequent symptom reporting among women with cosmetic breast implants. Plast Reconstr Surg 2001; 107:214-21.

9. Handel N, Cordray T, Gutierrez J, et al. A long-term study of outcomes, complications, and patient satisfaction with breast implants. Plast Reconstr Surg 2006; 117:757-67.

10. Tebbetts JB. Axillary endoscopic breast augmentation: processes derived from a 28-year experience to optimize outcomes. Plast Reconstr Surg 2006;118:53S-80S.

11. Momeni A, Padron NT, Bannasch H, et al. Endoscopic transaxillary subpectoral augmentation mammaplasty: a safe and predictable procedure. J Plast Reconstr Aesthet Surg 2006;59:1076-81.

12. Burden WR, Kelley PM. Endoscopic breast subpectoral augmentation for second-degree breast ptosis. Ann Plast Surg 2001;46:238-41.

13. Lindsey JT. The case against medial pectoral releases: a retrospective review of 315 primary breast augmentation patients. Ann Plast Surg 2004;52:253-6.

14. Tebbetts JB. Achieving a predictable 24-hour return to normal activities after breast augmentation: part II. patient preparation, refined surgical techniques, and instrumentation. Plast Reconstr Surg 2002;109:293-305.

15. Munhoz AM, Aldrighi C, Buschpiegel C, et al. The feasibility of sentinel lymph node detection in patients with previous transaxillary implant breast augmentation: preliminary results. Aesthetic Plast Surg 2005;29:163-8.

16. Barnsley GP, Sigurdson LJ, Barnsley SE. Textured surface breast implants in the prevention of capsular contracture among breast augmentation patients: a meta-analysis of randomized controlled trials. Plast Reconstr Surg 2006;117: 2182-90.

17. Jacobson JM, Gatti ME, Schaffner AD, et al. Effect of incision choice on outcomes in primary breast augmentation. Aesthet Surg J 2012;32:456-62.

18. Stutman RL, Codner M, Mahoney A, et al. Comparison of breast augmentation incisions and common complications. Aesthetic Plast Surg 2012;36:1096-104. 